\title{
TERMOELETTRICITÀ: DALL'ARCO DI ALESSANDRO VOLTA AI GENERATORI TERMOELETTRICI FERRO-LIQUIDI
}

\author{
Nota del s.c. ANDREY A. VARLAMOV (*), \\ SERGEI G. SHARAPOV $(* *)$, IOULIA CHIKINA $(* * *)$
}

(Adunanza del 12 novembre 2020)

SunTO. - Iniziamo questa breve rassegna dall'entusiasmante storia della termoelettricità in cui sono stati coinvolti i nomi come Luigi Galvani, Alessandro Volta, Thomas Seebeck, Walter Nernst, William Tompson, Georg Wilhelm Hegel e molti altri. Si passa quindi ai concetti principali nella descrizione dei fenomeni termoelettrici, si discutono le modalità di aumento della loro grandezza ed efficienza, si formulano i requisiti per i moderni materiali termoelettrici e si presentano applicazioni termoelettriche già disponibili.

$* * *$

ABSTRACT. - We start this concise review from the exciting history of thermoelectricity in which such names as Luigi Galvani, Alessandro Volta, Thomas Seebeck, Walter Nernst, William Tompson, Georg Wilhelm Hegel and many others have been involved. We then move on to the main concepts in description of thermoelectric phenomena, discuss how to increase their magnitude and efficiency, formulate the requirements for modern thermoelectric materials and present thermoelectric applications that are already available.

(*) CNR-SPIN, c / o DICII-Università di Roma Tor Vergata, Roma, Italy.

E-mail: andrey.varlamov@spin.cnr.it ; varlamov@ing.uniroma2.it

(**) Istituto Bogolyubov di Fisica Teorica, Accademia Nazionale delle Scienze dell'Ucraina, Kiev, Ucraina

${ }^{(* * *)}$ LIONS, NIMBE, CEA, CNRS, Université Paris-Saclay, CEA Saclay, Gif-sur-Yvette, Francia 
È difficile sorprendere una persona moderna con domande su cosa sia l'elettricità o il trasferimento di calore: ognuno ha una sua precisa conoscenza di tali argomenti, e motivi per essere più o meno informato. Alcuni degli interpellati sono teorici, si basano sullo studio della fisica, mentre la maggior parte magari ricordano la sensazione indimenticabile di una scossa elettrica nel processo di riparazione di una presa con la tensione sul pannello non scollegata oppure quella dal contatto accidentale con un ferro caldo. Siamo certi che un numero molto minore di lettori abbia minore familiarità con il fenomeno della termoelettricità. Nell'antica mitologia greca, i centauri occupano un ruolo importante: creature selvagge e mortali con la testa e il busto di un uomo sul corpo di un cavallo, abitanti delle montagne e dei boschetti della foresta. Il fenomeno della termoelettricità è simile a un centauro: qui la tensione elettrica tra i bordi del campione si verifica a causa del suo riscaldamento irregolare.

\section{Controversia tra Luigi Galvani e Alessandro Volta}

La storia della scoperta e del successivo studio della termoelettricità risale a più di due secoli fa e inizia con la disputa passata alla storia tra Luigi Galvani e Alessandro Volta. A metà del XVIII secolo, lo studio dei fenomeni elettrici, inclusa l'elettricità animale, come quella generata dai fulmini o dalle scintille, divenne un argomento popolare nella scienza. In considerazione di ciò, un professore dell'Università di Bologna, il fisiologo Luigi Galvani, iniziò a studiare cosa succede a una rana preparata quando viene attraversata da una corrente. A seguito di numerosi esperimenti, Galvani era convinto che ogni volta che toccava i nervi della rana con un conduttore attaccato a una macchina elettrica e ne estraeva una scintilla, la rana veniva colta da tremori convulsi. Quindi Galvani continuò i suoi esperimenti e trovò le stesse contrazioni quando i muscoli e i nervi della rana appena preparata fossero collegati con un arco bimetallico (Fig. 1). Un'altra circostanza sperimentale ha attirato così tanto l'attenzione di Galvani che egli lo cita espressamente nel suo lavoro: la contrazione dei muscoli della rana risulta essere molto più forte se l'arco metallico è composto in serie di due metalli diversi: ad esempio ferro e rame. Questi esperimenti portarono il famoso italiano alla conclusione che l'elettricità fosse caratteristica dell'animale stesso: dai nervi ai muscoli, un fluido sembra fluire e una catena si forma quando il vaso di Leida viene scaricato. 


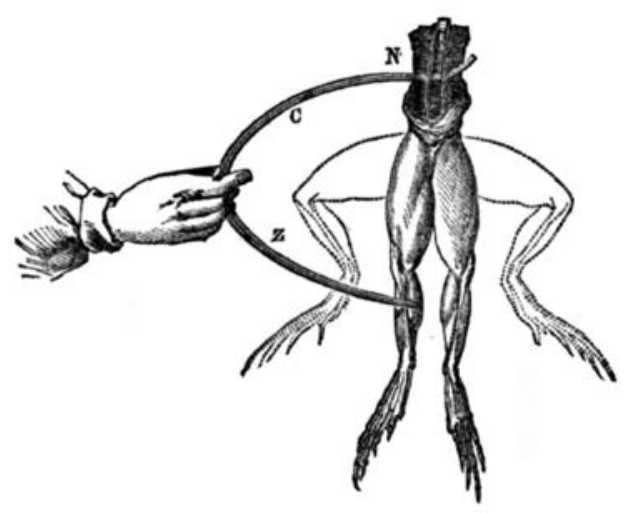

Fig. 1.

Alessandro Volta, professore all'Università di Pavia, era sospettoso della cosiddetta elettricità animale. Tuttavia, su insistenza dei suoi colleghi, ripete gli esperimenti descritti nell'opera di Galvani. Di conseguenza, il suo scetticismo iniziò a dissiparsi e il 3 aprile 1792 scrisse a Galvani: "Allora, eccomi finalmente convertito. Poiché io stesso sono diventato un testimone oculare e ho assistito a questi miracoli, forse sono passato dalla sfiducia al fanatismo" ${ }^{\text {(Fig. 2). In }}$ una conferenza pubblica del 5 maggio 1792, Volta parla degli esperimenti di Galvani, li esalta, ma allo stesso tempo esprime l'idea che una rana può essere solo un misuratore, un elettrometro, dieci volte più sensibile dell'elettrometro già esistente con foglie d'oro. Volta attira con insistenza l'attenzione su una caratteristica fisica degli esperimenti di Galvani: per indurre la contrazione muscolare, i metalli dell' arco devono essere diversi. Si chiede quindi: i conduttori metallici servono davvero solo a mettersi in contatto tra loro per stabilire una connessione che fornisce al fluido elettrico un percorso lungo il quale tende naturalmente a spostarsi da un luogo all'altro? I loro ruoli sono davvero puramente passivi o sono agenti attivi che mettono in moto una carica elettrica? Nei suoi ulteriori esperimenti, Volta

1 Volta plaude dapprima alla scoperta, che cerca di confermare ripetendo gli esperimenti. Le ripetute analisi e le rigorose misurazioni lo convincono che il disequilibrio elettrico è dovuto al contatto dei due metalli, la rana agendo come elettroscopio rivelatore: è inesatto affermare che nella rana si sia condensata dell'elettricità. 
mostra che il muscolo, in sostanza, non partecipa alla creazione del fenomeno stesso, la sua contrazione è l'effetto del flusso di una carica generata dal contatto di due metalli diversi. Così, infatti, scopre un fenomeno che è associato a una differenza di potenziale di contatto. Nei suoi dubbi, Volta va oltre. Come arco, cerca di usare un conduttore fatto di un metallo, ma posiziona le estremità del muscolo della rana e le estremità dell'arco in vasi con acqua calda e fredda. Il muscolo si contrae anche in questo esperimento, il che consente allo scienziato di spiegare il flusso del fluido elettrico (carica) mediante un riscaldamento irregolare del conduttore metallico (Fig. 3). È stato rilevato il collegamento tra corrente elettrica e differenza di temperatura! Descrive questi esperimenti il 10 febbraio 1794 nella sua lettera all'abate Vassalli [2].

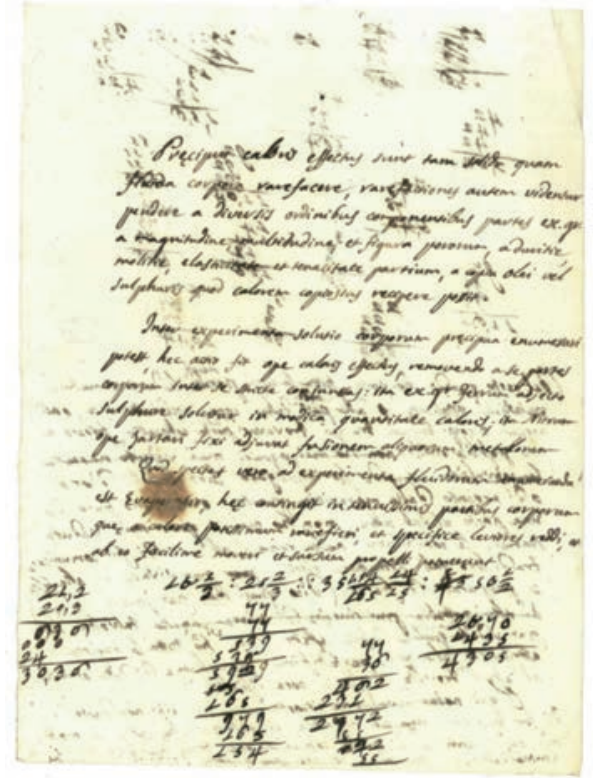

Fig. 2

Tuttavia, Volta non concentrò il suo ulteriore lavoro su questa scoperta: era più interessato all'applicazione pratica dell'effetto scoperto, a seguito di ulteriori ricerche egli creò una propria batteria, chiamata anche colonna voltaica [3-4]. 

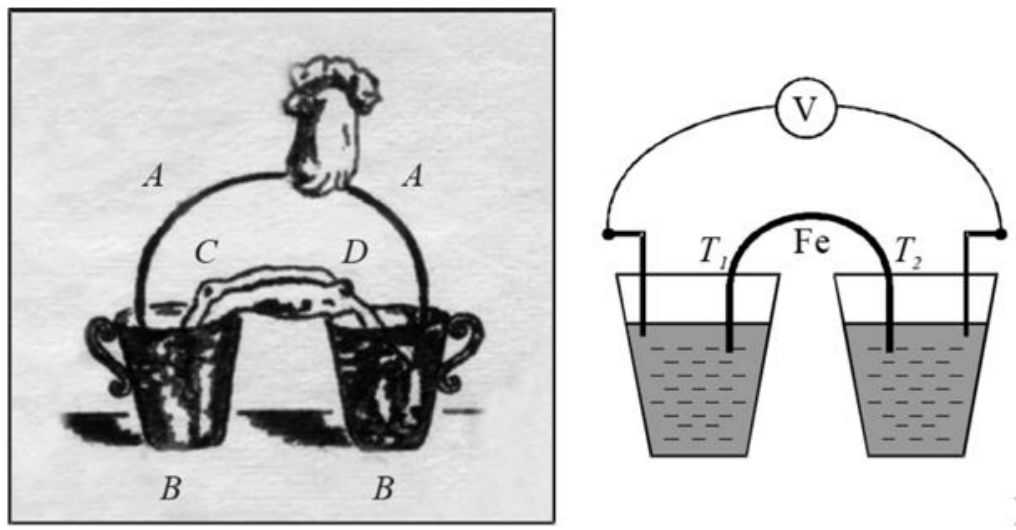

Fig. 3

\section{Jena SCHOOl E LA SCOPERTA Di JOHANN SEEBECK}

Thomas Johann Seebeck, fisico, chimico e medico tedesco-estone è nato a nel 1770 nella città anseatica di Revel (oggi Tallinn). All'età di 17 anni egli si trasferì in Germania per studiare medicina e nel 1792 superò ottimamente a Gottinga gli esami finali di medicina.

Tuttavia, sotto la forte influenza del carismatico professore di fisica Georg Christoph Lichtenberg, Seebeck decide di dedicarsi alla fisica. Nel marzo 1802 Seebeck ha conseguito il dottorato e si è trasferito a Jena. Qui si trova in un ambiente intellettuale ribollente la cui figura centrale è Johann Wolfgang Goethe, grande poeta, appassionato naturalista ed esploratore. In qualità di influente amministratore della corte di Weimar e amico personale del duca di Sassonia-Weimar-Eisenach, egli aiuta i giovani talenti ad avviare le loro carriere accademiche.

Seebeck incontrò per la prima volta Goethe a una cena il 3 dicembre 1803, dove incontrò anche il suo pari, il futuro famoso filosofo Hegel, con il quale strinse amicizia. Goethe sta cercando un assistente che lo aiuti nella sua ricerca ottica e Seebeck diventa per lui un'ottima scelta. La loro collaborazione a lungo termine ha portato al fatto che il contributo di Seebeck è diventato una parte importante, "Teoria dei colori" - il principale lavoro scientifico di Goethe [5]. Tuttavia, la vicinanza alla figura di Goethe lo travolge, lascia Jena. Infine, nel 1818, 
dopo una serie di vagabondaggi per le città universitarie della Germania, Seebeck fu eletto membro corrispondente dell'Accademia delle scienze di Berlino per il suo lavoro in ottica. Nel dicembre 1819 Seebeck scrive la sua ultima lettera a Goethe e finalmente si apre a nuove sfide.

Il 21 luglio 1820, Oersted pubblicò il suo famoso saggio "Esperimenti sull'effetto della corrente elettrica su un ago magnetico" [6], che apre le porte a un nuovo campo della scienza: l'elettromagnetismo. Seebeck lascia da parte i suoi esperimenti ottici e approfondisce le idee di Oersted. Ben presto, dopo diversi mesi di lavoro, Seebeck arrivò al principale risultato della sua carriera scientifica: la scoperta di un fenomeno che chiamò "termomagnetismo", per analogia con 1 “"elettromagnetismo" di Oersted.

Lo riferì in una riunione dell'Accademia di Berlino il 14 dicembre 1820 [7]. Due anni dopo, Oersted visitò il laboratorio di Seebeck a Berlino per conoscere i suoi esperimenti e, già nel marzo 1823, riferì della scoperta di Seebeck in una riunione dell'Accademia delle scienze francese. Allo stesso tempo, Oersted fornisce un'interpretazione fisicamente corretta di un nuovo fenomeno e introduce il termine attualmente accettato "termoelettricità" per la sua designazione. Oersted elogia il lavoro di Seebeck, definendolo "la più bella delle scoperte che sono nate dalla sua ricerca".

Una caratteristica quantitativa dell'effetto termoelettrico è il coefficiente di Seebeck - la differenza di potenziale tra le estremità del conduttore $\Delta \mathrm{V}$ che si verifica quando la differenza di temperatura $\Delta \mathrm{T}$ viene mantenuta tra di loro:

$$
S=-\frac{\Delta V}{\Delta T}=\frac{E}{\nabla T}
$$

dove $E=-\Delta V / \Delta x$ è l'entità del campo elettrico che si genera nel conduttore e $\nabla T=\Delta T / \Delta x$ è il gradiente di temperatura. Di conseguenza, la tensione generata da una termocoppia creata da due metalli diversi aventi temperature $T_{1}$ e $T_{2}$ è determinata dalla differenza tra le tensioni tra le loro estremità:

$$
V=S_{B}\left(T_{2}-T_{1}\right)-S_{A}\left(T_{2}-T_{1}\right)=\left(S_{B}-S_{A}\right)\left(T_{2}-T_{1}\right) .
$$


Sfortunatamente, nei metalli ordinari $V$, risulta essere molto piccolo (vedi Tab. 1. Si noti che i coefficienti di Seebeck per metalli diversi possono avere segni diversi). Ad esempio, quando una differenza di temperatura disponibile di $300^{\circ} \mathrm{C}$ viene applicata a una termocoppia, uno dei cui elettrodi è il bismuto, che occupa la posizione principale nella Tabella $\left(S_{B}=-73 \mu \mathrm{V} / \mathrm{K}\right)$, e il secondo è l'antimonio $\left(S_{A}=47\right.$ $\mu \mathrm{V} / \mathrm{K})$, la tensione generata è di circa $36 \mathrm{mV}$. Pertanto, per sostituire due batterie convenzionali tipo di quelle a dito utilizzate nei mouse per computer, nelle console e in molti altri gadget, sarebbe necessario collegare 100 di tali termocoppie e mantenere una differenza di temperatura di $300^{\circ} \mathrm{C}$ tra i loro elettrodi! Troppo!

Tab. 1.

\begin{tabular}{|c|c|c|c|}
\hline Metallo & $\begin{array}{l}\text { Coefficiente Seebeck } \\
(\mu \mathrm{V} / \mathrm{K})\end{array}$ & Metallo & $\begin{array}{l}\text { Coefficiente Metal Seebeck } \\
(\mu \mathrm{V} / \mathrm{K}) \text { (relativo al platino) }\end{array}$ \\
\hline Bismuto & -73.0 & Argento & +7.1 \\
\hline Nichel & -16.4 & Rame & +7.4 \\
\hline Palladio & -5.6 & Ferro & +16.0 \\
\hline Potassio & -9.0 & Antimonio & +47.0 \\
\hline
\end{tabular}

\section{L'UlTERIORE DESTINO DELLA SCOPERTA DI SEEBECK}

La termoelettricità ha trovato rapidamente la sua applicazione, almeno nei laboratori di fisica. Per esempio si consideri la nota legge di Ohm, scoperta e descritta da Ohm in due articoli, 1826 e 1827. Dopo la pubblicazione del primo articolo, a Ohm è fu consigliato di abbandonare la batteria galvanica, poiché la forza elettromotrice di tale sorgente è variata in modo significativo durante la misura. Risultati di misura più accurati (per il secondo articolo) furono ottenuti grazie all'utilizzo di una sola sorgente di corrente termoelettrica (coppia rame - bismuto), in grado di mantenere una tensione costante.

Ulteriori studi sul fenomeno scoperto da Seebeck continuarono negli anni ' 30 dell'Ottocento nelle opere di Peltier, dove fu scoperto l'effetto termoelettrico inverso: riscaldamento o raffreddamento del giunto di due conduttori quando una corrente elettrica scorre attraverso di esso. La sua essenza per diversi anni nel 1838 fu indagata da un fisico russo di origine tedesca, uno dei fondatori dell'ingegneria elettrica Heinrich Friedrich Emil Lenz). Egli mise una goccia d'acqua in una 
depressione alla giunzione di due aste di bismuto e antimonio (con resistenza R) e scoprì che quando una corrente elettrica scorreva in una direzione, la goccia si trasformava in ghiaccio e quando la direzione cambiava al contrario, il ghiaccio si è scioglieva. Questo semplice e impressionante esperimento ha mostrato che quando in due diversi metalli fluiscono correnti attraverso il contatto, oltre al noto calore Joule $I^{2} R$, viene rilasciato o assorbito calore aggiuntivo in proporzione diretta alla corrente. Quest'ultimo si chiama calore Peltier.

Diversi decenni dopo, alla fine degli anni Ottanta dell'Ottocento, nelle opere degli scienziati tedeschi Nernst ed Ettingshausen, furono scoperti vari fenomeni termomagnetici, dove, oltre alla differenza di temperatura, entrò in gioco anche un campo magnetico, ma la natura microscopica della termoelettricità è rimasta in questo caso poco chiara.

Tuttavia, per quanto riguarda invece la termoelettricità? Fino alla metà degli anni ' 30 del XX secolo, non vi era comprensione a livello microscopico della struttura dei metalli stessi. Per creare una teoria appropriata, era necessario almeno prima comprendere la struttura dei loro atomi costituenti, e il necessario apparato matematico e concettuale - la meccanica quantistica - fu formulato solo alla fine degli anni '20.

Comunque, a livello fenomenologico, la comprensione del fenomeno della termoelettricità è andata avanti. Nella seconda metà del XIX secolo, nelle opere di Gibbs, Boltzmann e altri scienziati, si sviluppò un nuovo campo della fisica: la meccanica statistica, che studia $i$ sistemi di un gran numero di particelle. La capacità di descrivere le loro proprietà usando la meccanica classica è illusoria: non è possibile non solo risolvere, ma neppure scrivere le equazioni della meccanica classica per un numero di particelle così elevato. Invece, gli scienziati stanno scoprendo modelli statistici completamente nuovi per tali casi. Capiscono che le informazioni extra (coordinate, velocità di tutte le particelle) semplicemente non sono necessarie per la adeguata descrizione delle proprietà richieste. Si dovrebbe in quel caso imparare a porre nuove domande alla Natura e capire come ottenere risposte.

Tra gli altri nuovi concetti, Gibbs nel 1875 introdusse il potenziale chimico $\mu$, l'energia che deve essere spesa per aggiungere un'altra particella al sistema di un gran numero di particelle.

In condizioni di equilibrio, questo valore rimane costante per tutto il volume del sistema, altrimenti particelle fluirebbero da un luogo all'altro. Il concetto di potenziale chimico può essere facilmente generalizzato al caso di un sistema che si trova in un campo esterno (con il 
quale, in quanto segue, si intende un campo elettrico di potenziale $\varphi$ ): per questo, al potenziale chimico $\mu$, basta aggiungere la corrispondente energia potenziale di una particella con una carica q da essa acquisita entrando nel sistema stesso. Ora la condizione di equilibrio diviene la costanza del potenziale elettrochimico $\mu+q \varphi$.

Torniamo all'esperienza di Alessandro Volta nel 1794. Mise le estremità opposte del conduttore di ferro in acqua bollente e ghiacciata, creando così un gradiente di temperatura nel sistema di elettroni liberi nel metallo. Questi elettroni, insieme al riscaldatore e al frigorifero, in senso stretto, non sono un sistema di equilibrio: per mantenere la differenza di temperatura, il calore deve essere continuamente fornito e rimosso ad esso. Tuttavia, se le estremità non sono collegate tra loro in un circuito elettrico (diciamo, da una zampa di rana), gli elettroni si muovono al primo momento in modo che la loro densità corrisponda alla temperatura locale (come qualsiasi gas a pressione costante: dove è più freddo, maggiore è la densità). Tuttavia, poiché sono particelle cariche, quindi, all'interno del metallo si genera un campo elettrico diverso da zero e una differenza di potenziale tra le estremità del conduttore. Ecco la spiegazione della termoelettricità, occorre solo sapere dell'esistenza di elettroni liberi nel metallo (l'elettrone stesso fu scoperto solo nel 1897).

Come si possono trasformare le considerazioni qualitative di cui sopra in una formula utile per il coefficiente di Seebeck? Bastano poche righe. Il potenziale elettrochimico nel conduttore rimane costante:

$$
\mu+q \varphi=\text { const. }
$$

Pertanto, la sua derivata rispetto alla coordinata (consideriamo il conduttore unidimensionale) è uguale a zero

$$
d \mu / d x+q d \varphi / d x=0
$$

L'ultima derivata non è altro che l'intensità del campo elettrico presa con il segno opposto: $E=-d \varphi / d x$. Quindi, arriviamo all'equazione

$$
d \mu / d x=q E .
$$

Il potenziale chimico ora cambia da punto a punto e dipende dal valore della temperatura locale. Pertanto, la sua derivata può essere calcolata prima differenziando rispetto alla temperatura, e successivamente differenziando quest'ultima rispetto alla coordinata: 


$$
d \mu / d x=(d \mu / d T)(d T / d x)=(d \mu / d T) \nabla T .
$$

In questo modo,

$$
(d \mu / d T) \nabla T=q E,
$$

e arriviamo alla cosiddetta formula di Kelvin, che collega il coefficiente di Seebeck alla derivata del potenziale chimico rispetto alla temperatura:

$$
S=\frac{E}{\nabla T}=\frac{1}{q}(d \mu / d T) \text {. }
$$

Diciamo sin d'ora che questa formula non descrive l'effetto termoelettrico in tutti $\mathrm{i}$ casi possibili, tuttavia sarà estremamente utile per la nostra ulteriore descrizione.

\section{Potenziale Chimico e coefFiciente di SeEbeck Del Metallo}

Come abbiamo già dimostrato, conoscendo il potenziale chimico, più precisamente, la sua dipendenza dalla temperatura, il coefficiente di Seebeck può essere trovato usando la formula di Kelvin. Il potenziale chimico di un gas elettronico degenere stesso si trova elementare: a temperature zero assoluto, quello coincide semplicemente con l'energia di Fermi. Infatti, se vogliamo aggiungere un altro elettrone al gas elettronico, ultimo dovrà essere messo alla superficie di Fermi, tutti gli altri posti già sono stati occupati anche durante la costruzione. In questo modo,

$$
\mu(T=0)=\epsilon_{F}=\frac{3^{2 / 3} \pi^{4 / 3} \hbar^{2}}{2 m} n^{2 / 3} .
$$

Un centimetro cubo di metallo contiene circa atomi, e se ciascuno di essi dona almeno un elettrone per uso generale, allora l'energia di Fermi sarà di circa $5 \cdot 10^{-19} \mathrm{~J}$, cioè circa $3 \mathrm{eV}$, che corrisponde ad una temperatura 5 volte superiore alla temperatura della superficie solare! Le velocità degli elettroni nello spazio della quantità di moto vicino alla superficie di Fermi sono enormi: sono percentuali della velocità della luce.

Tuttavia, questa conoscenza non ci basta: per trovare il coefficiente di Seebeck, il potenziale chimico deve essere differenziato rispetto alla temperatura. Si può ricavare che con l'aumentare della temperatura in un gas di Fermi degenere, cambia in modo quadratico: 


$$
\mu(T)=\epsilon_{F}-\frac{\pi^{2}}{6} \frac{\left(k_{B} T\right)^{2}}{\epsilon_{F}} .
$$

Quindi, usando la formula di Kelvin, troviamo il valore del coefficiente per un gas elettronico degenere

$$
S=-\frac{\pi^{2} k_{B}^{2}}{3 \mathrm{e}} \frac{T}{\epsilon_{F}}
$$

Da questa espressione diviene chiaro il perché l'effetto Seebeck è così debole nei metalli ordinari, dove, come abbiamo già visto, l'energia di Fermi è molto alta $\left(\epsilon_{F} \simeq 3 \mathrm{eV}\right)$. Quindi, per la temperatura ambiente, la nostra stima conduce a un valore per il coefficiente di Seebeck dell'ordine di alcuni microvolt per Kelvin, che rende i metalli inadatti all'uso come generatori di tensione termica.

\section{EFFETTO SEEBECK NEI SEMICONDUTTORI}

Esistono stime che mostrano che il costo dell'elettricità generata all'inizio del XX secolo dalla combustione diretta del combustibile in una caldaia a vapore e dalla conversione della pressione del vapore in lavoro meccanico era quasi 40 volte inferiore al costo dell'elettricità generata da fonti termoelettriche disponibili a quel momento storico [8]. E solo nel 1929, sulla base del rilevamento sperimentale di valori elevati (centinaia di microvolt per Kelvin) del coefficiente di Seebeck nei semiconduttori, Abram Ioffe avanzò l'idea che per aumentare l'efficienza dei termoelementi, i metalli dovrebbero essere sostituiti con semiconduttori. È possibile capire perché i semiconduttori sono materiali più promettenti per le fonti di energia termoelettrica come segue. Nel valutare il coefficiente di Seebeck nei metalli, abbiamo utilizzato il fatto che il numero di elettroni non cambia. Nei semiconduttori questo non è il caso e la concentrazione dei portatori di carica dipende molto (in modo esponenziale) dalla temperatura, portando ad una dipendenza molto più forte del potenziale chimico da essa. Questa è la ragione per cui si ottengono elevati valori del coefficiente di Seebeck nei semiconduttori.

Tali valori sono particolarmente grandi nei cosiddetti semiconduttori drogati, che sono simili ai metalli discussi sopra. Per modificare le proprietà elettriche, vengono aggiunte appositamente delle impurità che, essendo incorporate nel reticolo cristallino, liberano (o sottraggo- 
no) gli elettroni in eccesso dal sistema. Di conseguenza, in un tale semiconduttore, proprio come abbiamo già visto con l'esempio di un metallo, si forma un gas di elettroni liberi. Poiché la concentrazione di impurità è sempre piccola rispetto alla concentrazione di atomi del semiconduttore stesso, anche l'energia di Fermi corrispondente è piccola. A temperature sufficientemente basse (e potrebbero essere temperature ambiente), questo gas risulta essere degenere e per il corrispondente coefficiente di Seebeck di un semiconduttore si può usare la formula (5), solo l'energia di Fermi in esso non sarà elettronvolt, ma solo decine o centinaia di millielettronvolt. Di conseguenza, il coefficiente di Seebeck aumenterà di decine o centinaia di volte rispetto ai suoi valori nei metalli (vedi Tab. 2).

Tab. 2.

\begin{tabular}{llc}
\hline Sostanza & Proprietà elettriche & Coefficiente di Seebeck $(\boldsymbol{\mu V} / \mathrm{K})$ \\
\hline Bismuto & Metallo & -73 \\
\hline Costantana & Metallo & -35 \\
\hline Nichel & Metallo & -15 \\
\hline Platino & Metallo & 0 \\
\hline Alluminio & Metallo & 3.5 \\
\hline Germanio & Semiconduttore & 300 \\
\hline Silicio & Semiconduttore & 440 \\
\hline Tellurio & Semiconduttore & 500 \\
\hline Selenio & Semiconduttore & 900 \\
\hline
\end{tabular}

IL PROBLEMA DELL'EFFICIENZA DI UN GENERATORE TERMOELETTRICO E MODALITÀ DEL SUO AUMENTO

L'efficienza di un dispositivo termoelettrico è determinata dal rapporto tra l'energia Joule trasmessa al carico e l'energia termica totale assorbita dal contatto nello stesso tempo. Di conseguenza, risulta essere direttamente correlato alla differenza di temperatura relativa e alle proprietà di trasporto del termoelettrico:

$$
\eta=Z T\left(\frac{\Delta T}{T}\right)
$$

Indicatore di qualità adimensionale $Z T=\sigma \cdot S^{2} / \kappa$ (dove $\sigma$ è la conducibilità elettrica del contatto, $\kappa$ è la sua conducibilità termica) carat- 
terizza l'efficienza del dispositivo termoelettrico e il numero $Z$ stesso è chiamato la figura termoelettrica di merito del materiale, ovvero il numero di Ioffe [9]. Purtroppo per i metalli l'indicatore di qualità risulta essere molto piccolo: alle temperature di esercizio caratteristiche dei dispositivi termoelettrici è dell'ordine di $10^{-3} \div 10^{-4}$.

$\grave{E}$ chiaro che per aumentare l'efficienza di un dispositivo termoelettrico, si dovrebbe ridurre la conducibilità termica del materiale utilizzato aumentandone la conducibilità elettrica e il coefficiente di Seebeck. Come abbiamo già osservato, quest'ultima nei metalli è molto piccola a causa dell'enorme concentrazione di elettroni e, di conseguenza, dell'elevata energia di Fermi. Ma gli elettroni forniscono anche al metallo un'elevata conduttività. È vero, come tutti sanno chi ha mai mescolato lo zucchero nel tè con un cucchiaio d'argento e si è scottato che il metallo ha anche un'elevata conduttività termica. La conducibilità del metallo è strettamente correlata al contributo elettronico alla conducibilità termica $\kappa_{-}$e dalla cosiddetta legge di Wiedemann-Franz (Fig. 4).

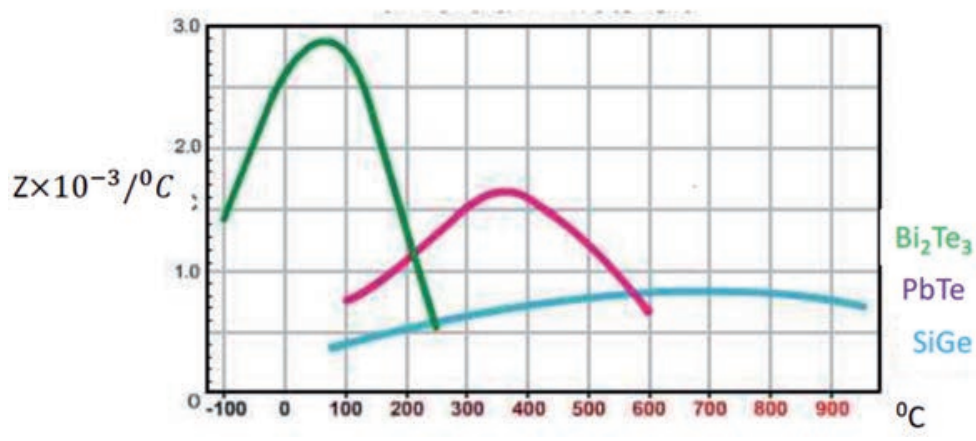

Fig. 4.

Tuttavia, oltre agli elettroni, anche il calore nel metallo viene trasferito dal reticolo. Il suo contributo a temperatura ambiente può essere molto significativo, quindi l'efficienza include la somma di entrambe le conduttività termiche. Pertanto, un aumento dell'efficienza di un dispositivo termoelettrico si riduce in pratica a:

- aumentare il coefficiente di Seebeck del materiale;

- un aumento della sua conducibilità;

- soppressione della sua conduttività termica reticolare.

Affinché i dispositivi termoelettrici diventino competitivi rispetto 
ad altre tecnologie di fonti di energia rinnovabile (ad esempio, solare e geotermica), è necessario raggiungere valori di efficienza $Z T \gtrsim 4$. Per raggiungere questo obiettivo sono stati spesi molti sforzi di ricerca. Di seguito presenteremo diversi esempi dei risultati di tali lavori.

\section{NUOVI MATERIALI PER LA TERMOELETTRICITÀ}

\section{a) Materiali termoelettrici con struttura dei difetti "panoscopica"}

La diminuzione della conducibilità termica è in gran parte dovuta alla struttura del materiale. Il reticolo trasferisce il calore per mezzo di onde elastiche che si propagano lungo di esso. Queste onde devono essere disperse, proprio come i frangiflutti riflettono le onde delle tempeste al largo delle coste dei mari e degli oceani. Il problema con la conduttività termica è che è necessario dissipare le vibrazioni del reticolo a tutte le scale, da quella atomica a quella micron. Ciò può essere ottenuto nanostrutturando elementi termoelettrici sfusi di nuova generazione introducendo in essi diffusori di vibrazioni reticolari su scala diversa. Questo uso "panoscopico" di difetti microstrutturali potenzia la diffusione di onde elastiche a diverse lunghezze d'onda senza sopprimere la conduttività elettronica, il che consente di creare materiali termoelettrici con elevate caratteristiche. Nei materiali termoelettrici nanostrutturati, le onde di trasferimento del calore con brevi lunghezze possono essere disperse da nanometri incorporati nel reticolo, mentre le onde a lunghezza d'onda lunga sono diffuse da difetti di gamma micron con un'architettura calibrata con precisione. Le strategie per il miglioramento dei materiali termoelettrici sono rivolte sia ai moderni materiali sfusi sia all'uso di sistemi a bassa dimensionalità. La nanostrutturazione di materiali termoelettrici a base di semiconduttori, che riduce la conduttività termica del reticolo con un aumento del coefficiente di Seebeck, ha portato a notevoli miglioramenti nella conversione dell'energia termica in energia elettrica. Tuttavia, anche i materiali più "promettenti" non possono ancora superare il requisito minimo di $Z T=4$ (vedi Tab. 3). Inoltre, i materiali nanostrutturati fino ad oggi realizzati presentano una serie di svantaggi tecnologici, ambientali ed economici,

2 Architettura gerarchica su tutte le scale. 
come le loro dimensioni limitate, i notevoli costi di produzione e il contenuto di materiali rari e tossici.

Tab. 3 .

\begin{tabular}{|c|c|c|}
\hline materiali & $Z T$ & Commenti \\
\hline $\begin{array}{l}\text { Ossidi laminati } \\
\left(\mathrm{SrTiO}_{3}\right)_{\mathrm{n}}(\mathrm{SrO})_{\mathrm{m}}\end{array}$ & $\sim 0.34 \mathrm{a} 1000 \mathrm{~K}$ & $\begin{array}{l}\text { Molto promettente per } \\
\text { applicazioni ad alte temperature }\end{array}$ \\
\hline $\begin{array}{l}\text { Calcogenuri di bismuto } \\
\left(\mathrm{Bi}_{2} \mathrm{Te}_{3}, \mathrm{Bi}_{2} \mathrm{Se}_{3}\right)\end{array}$ & $\begin{array}{l}\sim 0.8-1.0 \mathrm{a} \\
\text { temperatura ambiente }\end{array}$ & $\begin{array}{l}\text { ZT indipendente dalla } \\
\text { temperatura }\end{array}$ \\
\hline $\begin{array}{l}\text { Calcogenuri di bismuto } \\
\text { nanostrutturati } \\
\text { (трехслойные } \mathrm{Bi}_{2} \mathrm{Te}_{3}, \mathrm{Bi}_{2} \mathrm{Se}_{3} \text { ) }\end{array}$ & $\begin{array}{l}\sim 2.4 \text { a temperatura } \\
\text { ambiente }\end{array}$ & Elevata conducibilità elettrica \\
\hline Leghe di silicio-germanio & $\begin{array}{l}\sim 0.7 \text { a temperatura } \\
\text { ambiente }\end{array}$ & $\begin{array}{l}\text { I migliori materiali termoelettrici } \\
\text { a temperature di } \sim 1000 \mathrm{~K}\end{array}$ \\
\hline
\end{tabular}

\section{TERMOELETTRICITÀ NEGLI ELETTROLITI E NEI LIQUIDI FERROMAGNETICI}

Gli scienziati continuano a cercare nuovi tipi di materiali termoelettrici ecologici ed economici, come polimeri, conduttori ionici, ecc. Uno di questi oggetti è recentemente stato trovato negli elettroliti liquidi. Risulta che i valori del coefficiente di Seebeck per tali sistemi sono solitamente di un ordine di grandezza maggiore $(S \approx 0.5 \mathrm{mV} / \mathrm{K})$ rispetto ai materiali semiconduttori, anche nanostrutturati. Inoltre, sono costituiti da elementi economici e non tossici. Sfortunatamente, la conduttività elettrica di tali liquidi è di diversi ordini di grandezza inferiore a quella dei semiconduttori drogati; pertanto, gli elettroliti liquidi erano considerati inefficaci per l'utilizzo del calore dissipato.

Di recente, la ricerca di liquidi ionici ha dato un nuovo impulso alla ricerca di materiali termoelettrici adatti ad applicazioni pratiche [10]. Questi ultimi sono i sali fusi, liquidi sia a temperatura ambiente sia a temperature ben superiori a $100^{\circ} \mathrm{C}$ (alcuni possono superare $\mathrm{i}$ $300^{\circ} \mathrm{C}$ ). A differenza degli elettroliti liquidi, insieme ai grandi coefficienti di Seebeck, i liquidi ionici hanno un'elevata conduttività elettrica, che garantisce la loro alta efficienza $(Z T \approx 2)$. Ad oggi, il valore più alto del coefficiente di Seebeck ottenuto in un ampio intervallo di temperatura in un sistema con un liquido ionico supera i $2 \mathrm{mV} / \mathrm{K}$. Pertanto, $\mathrm{i}$ liquidi ionici sono candidati promettenti per utilizzare le perdite di 
calore, ad esempio convertendo il calore del tubo di scarico di un'auto in elettricità, che sarà sufficiente per il funzionamento di un condizionatore d'aria che raffredda il suo interno.

Il coefficiente di Seebeck degli elettroliti liquidi può anche essere aumentato introducendo nanoparticelle magnetiche (ferrofluido) nel loro volume. La loro lenta deriva sotto l'influenza delle differenze di temperatura e l'interazione con gli elettrodi migliorano le proprietà termoelettriche del liquido. La comprensione esistente della natura termoelettrochimica dei liquidi complessi è ancora lontana dall'essere completa, tuttavia, in questo entusiasmante settore, è in corso una ricerca sperimentale e teorica attiva, promettendo applicazioni tanto attese nell'utilizzo di perdite di calore che non solo danneggiano l'economico, $\mathrm{ma}$ anche l'ecologia del nostro ambiente. I suddetti ferrofluidi sono un mezzo di lavoro per una nuova generazione di termogeneratori, che sono attualmente in fase di sviluppo da scienziati di un consorzio di diversi laboratori europei con ingegneri FIAT.

\section{QUALCHE PAROLA SULLE APPLICAZIONI}

Attualmente, i dispositivi termoelettrici hanno una vasta gamma di applicazioni che vanno dalla generazione di energia ai rivelatori di radiazioni termoelettriche. L'affidabilità e la semplicità dei dispositivi termoelettrici ne consente l'utilizzo anche quando le fonti energetiche tradizionali sono più efficienti. Oltre alle termocoppie, sono ampiamente utilizzati dispositivi che funzionano secondo il principio dell'effetto Peltier: refrigeratori per vari prodotti, che vanno dall'optoelettronica, ai piccoli frigoriferi e ai sistemi di raffreddamento / riscaldamento dei sedili delle automobili. Come materiale di lavoro in tali dispositivi, di norma viene utilizzata una coppia di semiconduttori $\mathrm{Bi}_{2} \mathrm{Te}_{3}-\mathrm{Sb}_{2} \mathrm{Te}_{3}$.

Jules Verne, nel suo romanzo "Ventimila leghe sotto i mari", pubblicato nel 1870, discusse la possibilità che il Nautilus potesse essere alimentato da una batteria termoelettrica alimentata dalle differenze di temperatura dell'acqua a diverse profondità. I moderni sottomarini nucleari, come sappiamo, utilizzano turbine a vapore più tradizionali. Ma sui veicoli spaziali, che vengono inviati nelle profondità dello spazio, ci sono davvero fonti di energia termoelettrica. Il fatto è che al di fuori del pianeta Marte, la luce solare è troppo debole per alimentare 
un veicolo spaziale a energia solare. Pertanto, l'energia elettrica necessaria per il funzionamento dei sistemi di bordo viene fornita convertendo il calore rilasciato durante il decadimento radioattivo di Pu238 in elettricità utilizzando coppie termoelettriche. Tali generatori termoelettrici a radioisotopi sono stati utilizzati dalla NASA in missioni come Apollo, Pioneer, Viking, Voyager, Galileo e Cassini. Gli alimentatori di Voyager senza parti mobili funzionano ancora, consentendo alla navicella di fare scoperte scientifiche dopo oltre 35 anni di funzionamento. Sul "Perseverance", che ha recentemente iniziato a studiare Marte, è installato anche un generatore termoelettrico a radioisotopo del peso di $45 \mathrm{~kg}$, che fornisce una potenza di 110 watt.

\section{RINGRAZIAMENTI}

Gli autori ringraziano il professor Attilio Rigamonti per le tante discussioni e consigli. A.V. e I. Ch. ringraziano l'appoggio del Progetto Europeo MAGENTA HORIZON 2020 (progetto n. 686767). Il lavoro di S.G. Sh. è stato in parte sostenuto dalla Programma di ricerca scientifica del Ministero dell'Istruzione e della Scienza Ucraina (MESU) e Ministero della Scienza e della Tecnologia dello Stato di Israele (MOST) e dall'Accademia Nazionale delle scienze dell'Ucraina (progetto n. 0117U000236).

\section{BIBLIOGRAFIA}

1. Alessandro Volta, "Sopra l'Elettricità Animale". Copyright: (images) Università degli Studi di Pavia, 1792.

2. Alessandro Volta, "Nuova Memoria sull'Eletricità Animale". Lettera Seconda del Don Alessandro Volta al Don Anton Maria Vassali, 1794.

3. L.I. Anatychuk, "Seebek or Volta?" Journal of Thermoelectricity. N1. - 1994. P. 9-10.

4. G. Pastorino, "Alessandro Volta and his role in thermoelectricity", Journal of Thermoelectricity 1-2009, p. 7.

5. J.W. Goethe, "Zur Farbenlebre". Cotta, Tubingen, 1810.

6. H.C. Oersted, "Experimenta circa effectum conflictus electrici in acum magneticam". Hafniae, 1820.

7. T.J. Seebeck, "Magnetische Polarisation der Metalle und Erze durch TemperaturDifferenz", Abhandlungen der Königlichen Akademie der Wissenschaften in Berlin, Abhandlungen von 1820-21, 1822, p. 289-346. 
8. A.A. Buryak N.B. Karpova, "Essays on thermoelectricity development", Naukova Dumka, 1988. -280 p.

9. M.V. Vedernikov and E.K. Iordanishvili, "A. F. Ioffe and origin of modern semiconductor thermoelectric energy conversion". 17th Int. Conf. on Thermoelectrics vol 1, pp. 37-42 (1998).

10. A.F. Ioffe, "Semiconductor Thermoelements and Thermoelectric Cooling". Infosearch, Limited, 1957.

11. S. Nakamae, "Magnetic Fluids for Thermoelectricity" in "New Trends in Nanoparticle Magnetism", Edited by Davide Peddis, Sara Laureti, Dino Fiorani, Springer Series in Materials Science 308, 2021. 